Features for Art Painting Classification based on Vector Quantization of MPEG-7 Descriptors

Non Peer-reviewed author version

IVANOVA, Krassimira; STANCHEV, Peter; VELIKOVA, Evgeniya; VANHOOF, Koen; DEPAIRE, Benoit; MITOV, lliya \& MARKOV, Krassimir (2011) Features for Art Painting Classification based on Vector Quantization of MPEG-7 Descriptors. In: Proceedings of the 2nd International Conference on Data Engineering and Management., p. 146-153..

Handle: http://hdl.handle.net/1942/11645 


\title{
Features for Art Painting Classification based on Vector Quantization of MPEG-7 Descriptors
}

\author{
Krassimira Ivanova ${ }^{1}$, Peter Stanchev ${ }^{1 \& 2}$, Evgeniya Velikova ${ }^{3}$, \\ Koen Vanhoof ${ }^{4}$, Benoit Depaire ${ }^{4}$, Iliya Mitov ${ }^{1}$, and Krassimir Markov ${ }^{1}$ \\ ${ }^{1}$ Institute of Mathematics and Informatics - Bulgarian Academy of Sciences, \\ Sofia, Bulgaria \\ \{kivanova@math.bas.bg, mitov@mail.bg, kkmarkov@math.bas.bg\} \\ ${ }^{2}$ Kettering University, Flint, MI, 48504, USA \\ \{pstanche@kettering.edu\} \\ ${ }^{3}$ Faculty of Mathematics and Informatics, Sofia University, Sofia, Bulgaria \\ \{velikova@fmi.uni-sofia.bg\} \\ ${ }^{4}$ IMOB, Hasselt University, Hasselt, Belgium \\ \{koen.vanhoof@uhasselt.be, benoit.depaire@uhasselt.be\}
}

\begin{abstract}
An approach for extracting higher-level visual features for art painting classification based on MPEG-7 descriptors is presented in this paper. The MPEG-7 descriptors give a good presentation of different types of visual features, but are complex structures. This prevents their direct use into standard classification algorithms and thus requires specific processing. Our approach consists of the following steps: (1) the images are tiled into non-overlapping rectangles to capture more detailed information; (2) the tiles of the images are clustered for each MPEG-7 descriptor; (3) vector quantization is used to assign a unique value to each tile, which corresponds to the number of the cluster where the tile belongs to, in order to reduce the dimensionality of the data. Finally, the significance of the attributes and the importance of the underlying MPEG-7 descriptors for class prediction in this domain are analyzed.
\end{abstract}

Keywords: Content-Based Image Retrieval (CBIR), Multimedia Semantics, Pattern Recognition, MPEG-7 Descriptors, Clustering, Vector Quantization, Categorization.

\section{Introduction}

The digitalized art painting space gives us the opportunity to immerse in the ocean of accumulated culture. Earlier we could only dream to see some masterpieces. Now our computer moves us to every chosen place and time. These abilities increase the expectations for easy resource discovery by different criteria. While one user could be interested in art paintings from a specific movement or artist, others would search for images with particular theme or composition, and still others can be attracted by some 
pure aesthetic influence of the image. Many efforts are aimed at combining text-based and content-based search technologies in real-world image retrieval.

MPEG-7 is an ISO/IEC standard developed by Moving Picture Experts Group. The formal name of MPEG-7 is "Multimedia Content Description Interface" (http://www.chiariglione.org/mpeg). The standard describes the multimedia content data that supports some degree of interpretation of the information meaning. MPEG-7 is not aimed at any application in particular. The elements that MPEG-7 standardizes support a broad range of possible applications. The visual descriptors in the MPEG-7 standard describe different aspects of the image content such as dominant colors, edginess, texture, etc. The MPEG-7 descriptors are often used in the process of image-to-image matching, searching for similarities, sketch queries, etc. [1].

In this paper we apply a vector quantization method on the MPEG-7 descriptors as a possible approach for dimensionality reduction. For obtaining simple visual characteristics we use a clustering procedure over the tiles. The processes of obtaining these attributes are realized in our laboratory for art-painting semantic image retrieval APICAS. The significance analysis of received characteristics is conducted in the data mining analysis environment PaGaNe [2]. The examination of the accuracy for predicting the artists' names or movements is made in the Waikato environment for Knowledge Analysis [3].

The rest of the paper is organized in the following way. In section 2 existing systems in the field of art painting image analysis are described. Section 3 presents our methodology for extracting visual features from MPEG-7 descriptors based on vector quantization. Section 4 describes the software used for our research. Section 5 presents the received results. Finally, some conclusions and future work directions are highlighted.

\section{Existing Art Image Analyzing Systems}

In the field of art-painting image retrieval, several systems propose a variety of approaches for defining high level visual characteristics. Some aspects of image content are used with an existing ontology of art space.

S. Kröner and A. Lattner [4] trained a naive Bayes classifier to distinguish among the artists. They use five measures: three of them measure the ratio of black and white pixels and two take the stroke direction. The method is evaluated on a data set of 60 drawings, containing 41 drawings by Delacroix and 19 drawings from other artists. They achieved correct classification for about $87 \%$ of the drawings.

A study of the personal style of artists in the database of the Austrian National Library is presented in [5]. In this research, a computer-aided classification and recognition system for portrait miniatures has been developed. It enables a semiautomatic classification based on brush strokes and the hierarchical structure of the classification steps allows a top-down classification. As for the face recognition, a bottom-up approach is used instead. The final goal is to reduce the number of potential candidate artists to a minimum. The extension of the system, presented in [6], uses a hierarchical database indexing method based on Principal Component Analysis. The description incorporates the eyes as the most salient region in the 
portraits. 586 portrait miniatures have been tested in order to determining the orientation of eyes. The overall accuracy was about $87 \%$.

D. Keren [7] proposed a framework for classification of paintings based on local features derived from coefficients of a discrete cosine transform. After calculating the local features, each pixel is classified and the overall classification of the image is determined from a majority vote of the pixel values. The experiments were made over the works of Rembrandt, Van Gogh, Picasso, Magritte, and Dali. The training set contained ten paintings from each painter and the test set consisted of twenty to thirty paintings for each painter. For the five painters tested, a "tournament scheme" classifier was implemented and the rate of success was $86 \%$.

The Art Historian system [8] utilizes an automatic extraction of features of paintings' art movements. It is shown that the feature set enables one to highlight art movements efficiently. In the classifier design, a statistical pattern recognition approach is exploited using Bayesian, k-NN and SVM classifiers. It has been shown that the art movements of paintings can be indexed by exploiting the following six measures: (1) Percentage of dark colors; (2) Gradient coefficient calculated from the gradient map of the painting image; (3) Number of local and global maxima in the luminance histogram; (4) Color range that the peak point of the luminance histogram corresponds to; (5) The deviation of average grey level acquired within each block from the average grey level acquired within the entire image after the partition of the painting image into identical blocks; (6) The deviation of grey level distribution from the Gauss distribution. In the given experiments, the training set was constructed with 27 original paintings that belong to classic, cubic or impressionist movements, 9 from each class. The six features were extracted for every image in each movement. In order to observe efficiency of the proposed feature set and robustness to changes in the illumination and resolution, a test set was created with lighting effects and resolution changes. The testing set included 107 paintings from the different movements taken into account. The overall classification accuracy varied from $81.66 \%$ to $91.66 \%$ depending of used dimensionality and classification method.

In Lightweight Image Retrieval System for Paintings [9], the image indexing features are divided into the following three groups: (1) Canvas features: max, min, mean, median, and standard deviation from each of the red, green, and blue color channels; (2) Color features: intensity mean (measures the global brightness of a grayscale image), color frequency distribution (measures the degree of disorder found in the frequency distribution of colors in a painting); (3) Edge characteristics: line count (uses the Sobel edge detector to identify lines in the image). The tests for identifying the work of individual painters were run over set of 100 training images, which contained 10 images from the corpus of 10 artists and a testing set of 90 images, randomly chosen from the work of these artists. In $81 \%$ of test cases, the system retrieved at least one painting by the same artist in the top ten closest matches, suggesting that the model is effective for interactive classification of paintings by the artist. Another test was based on a database of 500 images drawn from the Web Museum. Although the overall retrieval rate was only $49.2 \%$, the system performed particularly well with respect to certain artists, distinguishing $87.5 \%$ for Aertsen and Velazquez. An analysis of the classification errors reveals that the system is effectively classifying artistic style even when it fails to classify the artist correctly. 
The team, headed by R. Jain [10] uses annotation of paintings based on brushwork, where brushwork is modeled as part of the annotation of high-level artistic concepts such as the movements of artists using defined ontology of so cold "brushworks", based on specific combination of low-level feature values of gradient, texture, hue and light contrast, and homogeneity, which characterize some style of the painting. The experiments with 4880 patches from 30 paintings of Renaissance, Fauvism, Impressionism, Post-Impressionism, Expressionism and Pointillism painting styles, where $75 \%$ are used for training and 25\% for testing, show accuracies between $80 \%$ and $95 \%$ in variants, where multiple experts are used.

\section{Proposed Approach for Feature Extraction}

MPEG-7 descriptors are complex descriptors, which provide a good presentation of different types of visual features. The description of the structure of MPEG-7 descriptors and algorithms is given in [11].

These complex structures need specific processing and cannot be put directly into generic classification algorithms.

\subsection{Used MPEG-7 Descriptors}

Here we give a brief explanation of each MPEG-7 descriptor examined in our work and which values we use in further processing:

- Scalable Color (SC) represents the color histogram in the HSV color space, encoded by a Haar transform. For presenting the image or a selected part, Scalable Color needs a vector with 64 attributes;

- Color Layout (CL) specifies the spatial distribution of colors using YCbCr color space. We use the first quantized $D C T$ coefficient of the $Y, C b$ and $C r$ components, the next five successive quantized DCT coefficients of the $Y$ component and the next two quantized DCT coefficients of the $\mathrm{Cb}$ and $\mathrm{Cr}$ component. These coefficients are used to form three vectors, which contain all extracted values of the $Y, C b$ and $C r$ components:

$$
\begin{aligned}
& D Y=\left\{D Y_{y} ; y=1 . .6\right\} \quad D Y_{y}= \begin{cases}\text { YDCCoeff } & y=1 \\
Y_{\text {ACCoeff }} & y \neq 1\end{cases} \\
& D C b=\left\{D C b_{y} ; y=1 . .3\right\} D C b_{y}= \begin{cases}\text { YDCbCoeff } & y=1 \\
\text { YACbCoeff } & y \neq 1\end{cases} \\
& D C r=\left\{D C r_{y} ; y=1 . .3\right\} D C r_{y}= \begin{cases}\text { YDCrCoeff } & y=1 \\
\text { YACrCoeff } & y \neq 1\end{cases}
\end{aligned}
$$

Thus, the Color Layout vector has 12 attributes;

- Color Structure (CS), which specifies both color content and the structure of the content. The descriptor expresses local color structure in an image by means of a 
structuring element that is composed of several image samples. We use a vector with 64 attributes for representing the Color Structure;

- Dominant Color (DC). We reconfigured the presentation of this descriptor as three vectors, representing distribution of quantized hue, saturation and luminance. Such method is already precisely described and used by us in [12]. After this quantization we receive a vector with 23 attributes (13 for hue +5 for saturation +5 for luminance);

- Edge Histogram (EH) specifies the spatial distribution of five types of edges in local image regions (four directional edges - vertical, horizontal, 45 degree, 135 degree and one non-directional). Edge Histogram descriptor produces a vector with 80 attributes;

- Homogeneous Texture (HT) characterizes the region texture using the energy and energy deviation in a set of frequency channels. A vector with 60 attributes is used, presenting Energy and Energy Deviation.

As a result we obtain a vector, which when taken together results more than 300 attributes. First experiments with the image as a whole reveal that most classifiers could not produce a classification model with accuracies (see section 5) comparable with the systems described in sector 2. To improve the accuracies we adopted the representation by eliminating redundancy and by introducing locality.

Some of the MPEG-7 descriptors are alternative. For instance, Scalable Color, Color Layout, Color Structure and Dominant Color concern different aspects of the same phenomenon, i.e. distribution of the color within the image or region. It means that not all descriptors have to be given in the classification process. The first goal of this research is to examine which of them are more convenient to be used for extracting simple visual features for the purposes of class recognition.

Visual attributes can represent global characteristics, concerning whole images, or they can be extracted over the part of the images (specific region or tile of the image). Both approaches have their strengths: global attributes deliver integral temper of the image. Local attributes can capture more detailed information, which characterize the artists' styles or movements' specifics but introduce redundancy for the classifier. For reducing the computational weight and redundancy a possibility is to choose only part of the tiles - only chess ordered tiles, starting from the first tile $(1,1)$ or from the second one $(1,2)$ as well as taking into account only left sided or right sided tiles. In this paper we use all the tiles. The second goal of this research is to examine the trade of between the global and local approach.

\subsection{Vector Quantization Approach}

In our approach we split the images into $m \times n$ non-overlapping rectangles (tiles). The tiles are marked as $(i, j)$, where $i \in 1 \ldots m$ and $j \in 1$...n. Index $i$ increases from the left tile to the right tile and index $j$ increases from the top tile to the bottom tile of the image. 
Let $I=\left\{I_{p} \mid p=1 . . k\right\}$ be the observed set of $\mathrm{k}$ images. Each image is divided into non-overlapping $m \times n$ tiles and as a result a set of $k \times m \times n$ tiles $T=\left\{I_{p}^{i j} \mid p=1 \ldots k, i=1 \ldots m, j=1 \ldots n\right\}$ is produced.

From the observed set, a subset $L I=\left\{I_{q} \mid q=1 \ldots l, l<k, I_{q} \in I\right\}$ of representative images is extracted, which is used as learning set. The images of this subset produce a set of $l \times m \times n$ tiles $L T=\left\{I_{q}^{i j} \mid q=1 . . . l, i=1 \ldots m, j=1 \ldots n\right\}$.

For each MPEG-7 descriptor $X \in\{S C, C L, C S, D C, E H, H T\}$ the algorithm consists of following steps:

- for all tiles in $L T$, the MPEG-7 descriptor and the corresponding feature vector $X \in\{S C, C L, C S, D C, E H, H T\}$ is calculated;

- $\quad$ the tiles in $L T$ are clustered. We used "repeated bisections". The number of the clusters can be different for different MPEG-7 descriptors, but in this realization for all descriptors an equal number $\alpha$ is used;

- $\quad$ the centroids of each cluster are calculated;

- $\quad$ for each tile a value, which corresponds to the number of the cluster where the tile belongs to, is assigned;

- $\quad$ for tiles which were not in the learning set $I T \backslash L T$, the membership of their centroids is calculated using $L_{1}$-metric, and the number of the corresponding cluster is assigned as a value of the tile.

As a result, each image of $I$ is represented with a feature vector with $x \times m \times n$ numerical attributes, where $x$ is the number of MPEG-7 descriptors. For instance in case of using all MPEG-7 descriptors for $3 \times 3$ tiling, the number of attributes in this vector is $6 \times 3 \times 3=36$. In case of selecting only a subset of the available tiles (chess order or left/right side), the number of features reduces.

A specific of this approach is that received attributes are nominal. The main purpose of the prepared datasets after implementing this approach is to examine the significance of the attributes and the local/global trade-off for class prediction.

\section{Software Realization}

In order to realize the proposed algorithm, our laboratory for art-painting semantic image retrieval "Art Painting Image Color Aesthetic and Semantic" (APICAS), firstly presented in [13], was extended with additional functions.

For obtaining the MPEG-7 descriptors [11] our system refers to Multimedia Content Management System MILOS [14].

As clustering algorithm the program vcluster, which is part of the Cluto open source software package [15], is implemented in the system. CLUTO is used to cluster both low and high dimensional datasets and for analyzing the characteristics of the various clusters. As clustering method we have used the default method "repeated bisections". The similarity between objects is computed using the correlation coefficient. As clustering criterion function, cluster maximization of sum of square 
root of sums of similarities of vectors, belonging to given cluster, is used. In this method, each cluster is represented by its centroid vector and the goal is to find the clustering solution that maximizes the similarity between each vector and the centroid of the cluster to which it is assigned.

As knowledge analysis and testing environment, we used the data mining analysis environment PaGaNe [2]. We use PGN-1 classifier and implemented statistical analyzing tools for checking up our results and extracting regularities for artists' and movements' styles based on the extracted attributes.

\section{Experiments}

For our experiments we have used datasets that include 600 paintings of 18 artists from different movements of West-European fine arts and one group, which represent Orthodox Iconographic Style from Eastern Medieval Culture (Table 1). The pictures were obtained from different web-museum sources using ArtCyclopedia as a gate to the museum-quality fine art on the Internet as well as from different Eastern public virtual art galleries and museums for extracting Icons.

Table 1: List of the artists, which paintings were used in experiments, grouped by movements

\begin{tabular}{|l|l|}
\hline \hline Movement & Artist \\
\hline \hline Icons (60) & Icons (60) \\
\hline Renaissance (90) & Botticelli (30); Michelangelo (30); Raphael (30) \\
\hline Baroque (90) & Caravaggio (30); Rembrandt (30); Rubens (30) \\
\hline Romanticism (90) & Friedrich (30); Goya (30); Turner (30) \\
\hline Impressionism (90) & Monet (30); Pissarro (30); Sisley (30) \\
\hline Cubism (90) & Braque (30); Gris (30); Leger (30) \\
\hline Modern Art (90) & Klimt (30); Miro (30); Mucha (30) \\
\hline
\end{tabular}

We have made several experiments with different parameters for:

- $\quad$ the number of tiles - we have ran experiments with equal tiling over width and height from $1 \times 1$ (the whole images) up to $7 \times 7$ (i.e. $m=n \in\{1, \ldots, 7\}$ );

- $\quad$ the number of clusters - the experiments were carried out with 20, 40 and 60 clusters;

- $\quad$ as class value, we have used "artists' names" and "movements".

As the set of attributes we have used $\{X(i, j)\}$, where $i=1 \ldots m, j=1 . . . n$, $X$ is $S C, C L, C S, D C, E H, H T$.

We have processed the datasets under the procedures of attribute selection in order to receive the order of significance of attributes for prediction. We have implemented several attribute evaluation methods - Chi square; Filter; Information Gain, and they give almost equal results for ordering the significance of the attributes, diverging the order for some attributes only in 2-3 positions.

Later analyses are made using Chi square evaluation method. 
We have summarized the obtained order of attributes by types of descriptors and positions of the tiles by width, respectively by height.

\subsection{Evaluation of the attributes in respect to the type of underlying MPEG-7 descriptor}

Fig. 1 shows the distribution of significance of MPEG-7 descriptors for class prediction. As it is shown, the Color Structure (CS) descriptor is the most informative for our datasets.

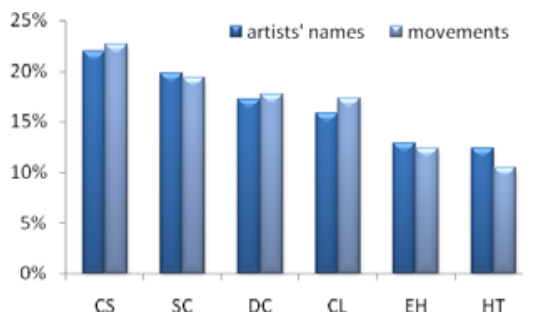

Figure 1. Average distribution of significance by MPEG-7 descriptors over datasets, using different tiling and clustering, using Chi square evaluation method

The dominance of the features, based on the color descriptors (CS, SC, DC, CL) is obvious, which leads to these assumptions:

- $\quad$ the artist's palettes, which are captured in color descriptors, are a powerful tool for creating the profiles of art painting images;

- $\quad$ using this approach, texture descriptors (EH, HT) can not produce sufficient quality attributes to present the specifics of the brushwork of the artists.

\subsection{Evaluation of the attributes in respect to the position of tiles}

The analysis of the significance of the tile position for the horizontal splitting (Fig. 2) and vertical splitting (Fig. 3), shows that the increasing the number of tiles reduces the differences between the zones.

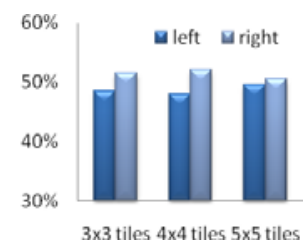

Figure 2. Distribution of significance of left side and right side of the images for $3 \times 3,4 \times 4$ and $5 \times 5$ tiling.

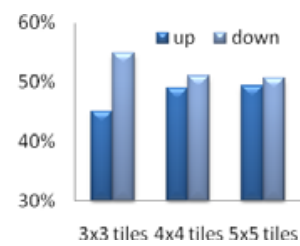

Figure 3. Distribution of significance of upper and lower zone of the images for $3 \times 3,4 \times 4$ and $5 \times 5$ tiling. 
Furthermore, we can examine the significance of the tiles in respect to the position in the images. Fig. 4 shows the distribution of significance of the tiles in respect to the horizontal position. The graphics show the distribution for $5 \times 5,6 \times 6$ and $7 \times 7$ tiling. As we can see, the differences are not distinctive, which means that horizontally each zone of the image is relatively equal important.

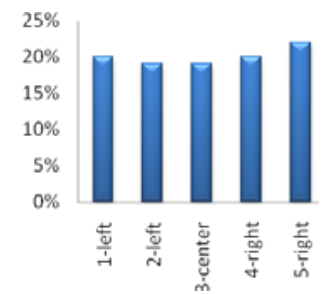

(a)

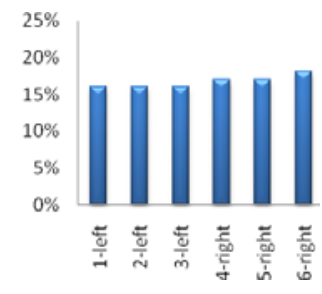

(b)

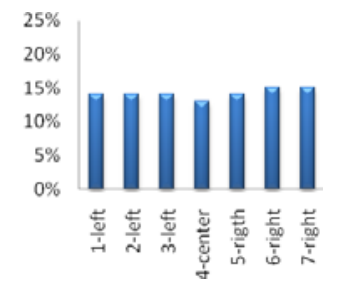

(c)

Figure 4. Distribution of significance of the tiles by position of width, $i \in 1$...m from left to right - (a) $m=5$; (b) $m=6$; (c) $m=7$.

The construction of many classical paintings is based on central symmetry. A little superiority of the right part of the image for a fewer number of tiling confirms the results from psychological theories for understanding human perception [16]. We attend to use this fact in further investigation with analyzing the tiles only of the right half of the image.

Similar analysis in respect to the vertical position of the tiles is shown on Fig. 5. Here, it becomes clear that outer tiles (and especially border tiles) are more informative (more distinctive for different classes) than inner tiles (and especially center tiles).

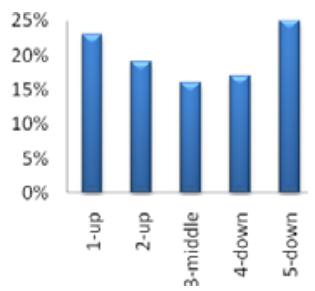

(a)

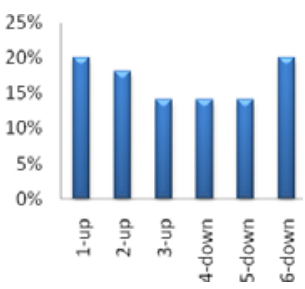

(b)

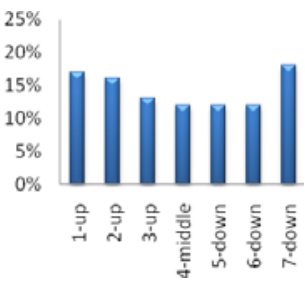

(c)

Figure 5. Distribution of significance of the tiles by position of height, $j \in 1$...n from up to down - (a) $n=5$; (b) $n=6$; (c) $n=7$.

This fact can also be explained with differences between constructions of composition in different styles [16]. While the central part of the image brings object or scene information, the borders are les burdened with this task. In order to supply the focus of the image, there are not usually specific objects found here, but only the ground patterns, which are specific for the artists or the school, in which the artists belongs. These patterns capture the ground of the artists' palette and brushwork. 


\subsection{Evaluation of the attributes in respect of the number of clusters}

Other experiments are focused on establishing the appropriate number of clusters in order to receive good classification results with lower computational cost. We have run ten-fold cross-validation over the datasets.

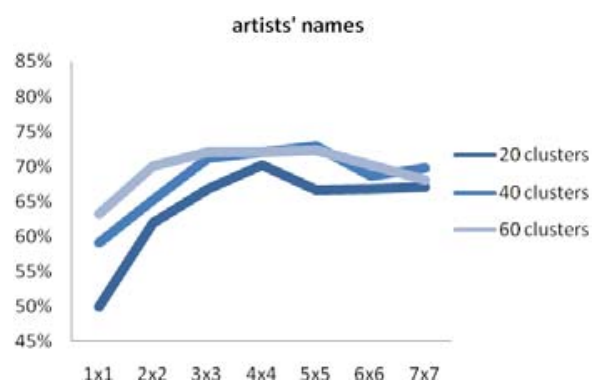

(a)

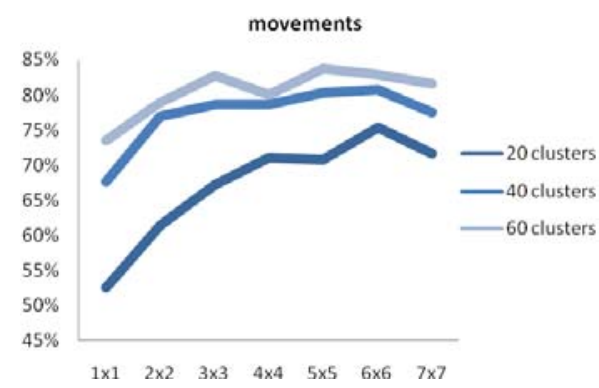

(b)

Figure 6. Classification accuracy for datasets with classes (a) artists and (b) movements using 20,40, 60 clusters and different numbers of tiling

The results displayed on Fig. 6, show that using vector quantization on MPEG-7 descriptors for the entire image (i.e. $1 \times 1$ tiling) is not so informative. Better but nonsufficient results are obtained for $2 \times 2$ tiling. Tiling $3 \times 3$ is the first with relatively good results. This result is also conceptually validated by the fact that $3 \times 3$ tiling corresponds to a rough approximation of the golden ratio, which usually lies in the compositions of the art paintings. The last observed tiling $7 \times 7$ shows a decreasing of accuracy, which can be explained by the fact that the pictures became too fragmented and the clusters fall not in the proper positions.

\subsection{Evaluation of the classification accuracy by different types of classifiers}

We have compared classification accuracy for different type of classifiers, realized in Waikato Environment for Knowledge Analysis (WEKA) [3] as well as with PGN-1 classifier, which is realized in our system PaGaNe [2]. All accuracies are based on ten-fold cross validation. Two datasets were used, which were both created with $4 \times 4$ tiling and 40 clusters. The first data set uses the artist's name as the class variable and the second data uses the artist's movement as the class variable.

We have aggregated some received results per group of classifiers as follows:

- Bayes (BayesNet, NaiveBayes, NaiveBayesSimple, NaiveBayesUpdateable, LBR);

- $\quad$ kNN (IB1, IBK, Kstar, HyperPipes, NNge);

- $\quad$ trees (ID3, J48, RandomTree, REPTree, SimpleCart, OLM);

- $\quad$ rules (DecisionTable, JRip, OneR, PART).

The rest of classifiers did not produce good results and we excluded them from the discussion. 


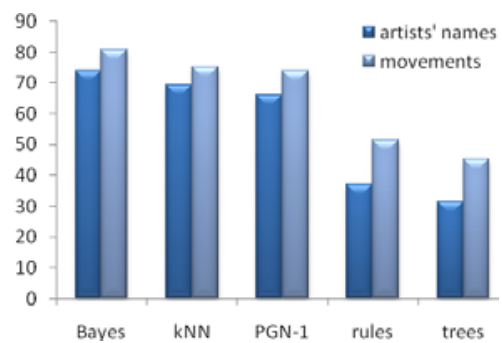

Figure 7: Average classification accuracy of Bayes, kNN, rules and trees classifiers and PGN-1 using ten-fold cross validation

On Fig. 7 we show the classification accuracy of the examined groups of classifiers in WEKA as well as the results from PGN-1. As we can see for these two datasets, Bayes and kNN classifiers give the best accuracy. Rules and trees show less satisfying results. This is not a surprise given that we retained only nominal attributes. PGN-1 gives good and stable results which are comparable with the best ones from WEKA. PGN-1 has the accuracy precision like kNN classifiers, but with priority to build the set of generalized non-contradictory association rules. We attend to use this property in further investigation, in order to search for definition of profiles of the artists or movements.

\subsection{Local analysis of the classification accuracy}

Table 2 gives the confusion matrix of PGN-1 over the second dataset, with the artist's movement as the class variable, with $4 \times 4$ tiles and 40 clusters. The rows indicate the class where the image actually belongs to. The columns show the class label assigned to the image by the classifier. The numbers of correctly recognized images of the corresponded class are represented on the diagonal.

Table 2: Confusion matrix of PGN-1 classifier over examining dataset with classes movements

\begin{tabular}{|r|c|c|c|c|c|c|c||c||}
\hline & ICON & $\begin{array}{c}\text { RENAIS- } \\
\text { SANCE }\end{array}$ & BAROQUE & $\begin{array}{c}\text { ROMAN- } \\
\text { TICISM }\end{array}$ & $\begin{array}{c}\text { IMPRES- } \\
\text { SIONISM }\end{array}$ & CUBISM & $\begin{array}{c}\text { MODERN } \\
\text { ART }\end{array}$ & Sum by row \\
\hline ICON & 17 & & & & & & 3 & 20 \\
\hline RENAISSANCE & & 21 & & 3 & 2 & 4 & & 30 \\
\hline BAROQUE & 1 & & 28 & & 1 & & & 30 \\
\hline ROMANTICISM & & 1 & 1 & 21 & 5 & & 2 & 30 \\
\hline IMPRESSIONISM & 1 & 1 & & 1 & 24 & 1 & 2 & 30 \\
\hline CUBISM & & 2 & 2 & 3 & 1 & 19 & 3 & 30 \\
\hline MODERN ART & 2 & 1 & 1 & & 1 & 1 & 24 & 30 \\
\hline \hline Sum by column & 21 & 26 & 32 & 28 & 34 & 25 & 34 & 200 \\
\hline \hline
\end{tabular}

On the base of this matrix, Precision, Recall and F-measure are calculated as follows: 
- Precision (Figure 8a) is the number of correct answers over the number of the images, belonging to the corresponded class (diagonal value over the sum by row);

- Recall (Figure 8b) is the number of correct answers over the total number of the images assigned to this class by classifier (diagonal value over the sum by column);

- F-measure (Figure 8c) is calculated as a harmonic mean of precision and recall: $F=2 \cdot \frac{\text { precision } \cdot \text { recall }}{\text { precision }+ \text { recall }}$

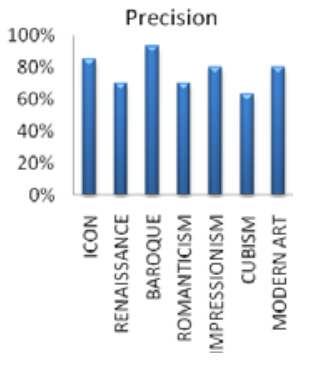

(a)

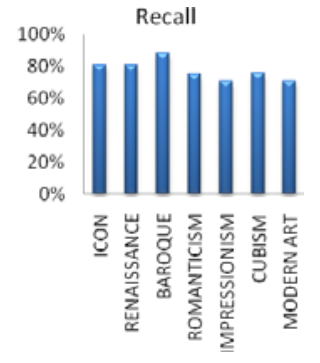

(b)

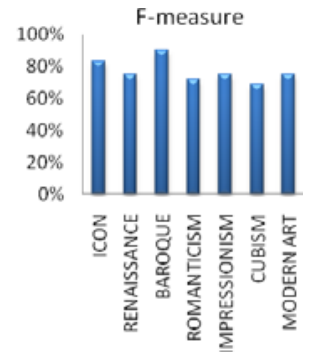

(c)

Figure 8. (a) Precision, (b) Recall and (c) F-measure for the examining set with classes movements

The results presented in Fig. 8, shows that the classification accuracy for some movements (for instance, Baroque) is very good. The nature of a part of paintings from Romanticism, presenting the landscapes [17], tends to confuse with similar paintings from Impressionism. Our expectation that Impressionism and Modern art with their different brushwork techniques will be distinctively separated by attributes, based on vector quantization of Homogeneous Texture or Edge Histogram descriptors were not confirmed, which means that for capturing brushwork specifics we have to use another approach or to find different kind of features.

\section{Conclusions}

In this article we have presented an approach for analyzing visual characteristics, based on MPEG-7 descriptors. The method allows on one hand tiling and on the other hand a huge reduction of the dimensionality of the data. For the used data sets, $4 \times 4$ tiling and 40 clusters seemed optimal. From the analysis we learned that the artist's palettes, which are captured in color descriptors, are a powerful tool for creating the profiles of art painting images. The texture descriptors $(E H, H T)$, in our specific presentation, can not produce sufficient quality attributes to present the specifics of the brushwork of the artists. Position analysis confirmed other research results.

In further research, we will look for combinations of characteristics in order to form profiles for artists or movements. 


\section{Acknowledgments}

This work was supported in part by Hasselt University under the Project R-1875 "Search in Art Image Collections Based on Color Semantics" and by the Bulgarian National Science Fund under the Project D002-308/19.12.2008 "Automated Metadata Generating for e-Documents Specifications and Standards".

\section{References}

1. Stanchev, P., Green Jr., D., Dimitrov, B.: Some Issues in the Art Image Database Systems. Journal of Digital Information Management, Volume 4, Issue 4, December 2006, pp. 227-232 (2006)

2. Mitov, I., Ivanova, K., Markov, K., Velychko, V., Vanhoof, K., Stanchev, P.: PaGaNe - a Classification Machine Learning System Based on the Multidimensional Numbered Information Spaces. Fourth Int. Conf. Intelligent Systems and Knowledge Engineering (ISKE), 27-28.11.2009, Hasselt, Belgium, Printed in World Scientific Proceedings Series on Computer Engineering and Information Science, No:2, pp. 279-286 (2009)

3. Witten, I., Frank, E.: Data Mining: Practical Machine Learning Tools and Techniques. 2nd Edition, Morgan Kaufmann, San Francisco (2005)

4. Kröner, S., Lattner. A.: Authentication of Free Hand Drawings by Pattern Recognition Methods. Proc. of the 14th International Conference on Pattern Recognition, Brisbane, Australia, pp. 462-464 (1998)

5. Sablatnig, R., Kammerer, P., Zolda, E.: Hierarchical Classification of Paintings Using Face- and Brush Stroke Models. Proc. of the 14th International Conference on Pattern Recognition (ICPR), 16-20.08.1998, Volume 1, IEEE Computer Society, Washington, DC, 172 (1998)

6. Saraceno, C., Reiter, M., Kammerer, R., Zolda, E., Kropatsch, W.: Pictorial Portrait Indexing Using View-based Eigen-eyes. D. Huijsmans and A. Smeulders (eds), Visual Information and Information Systems, Lecture Notes in Computer Science, vol. 1614, pp. 649-656 (1999)

7. Keren, D.: Painter Identification Using Local Features and Naive Bayes. Proc. of the 16th International Conference on Pattern Recognition, Quebec, Canada, Volume 2, pp. 474-477 (2002)

8. Icoglu, O., Bilge, G., Sanem, S.: Classification and Indexing of Paintings Based on Art Movements. 12th European Signal Processing Conference (Eusipco), 6-10.09.2004, Vienna Austria, pp. 749-752 (2004)

9. Lombardi, T., Sung-Hyuk, C., Tappert, C. A Lightweight Image Retrieval System for Paintings. Proceedings of SPIE - Volume: Storage and Retrieval Methods and Applications for Multimedia, 5682, pp. 236-246 (2005)

10. Marchenko, Y., Chua, T., Jain, R.: Semi-supervised Annotation of Brushwork in Painting Domain Using Serial Combinations of Multiple Experts. Technical Report, NUS, Singapore (2006)

11. International Standard ISO/IEC 15938-3 Multimedia Content Description Interface Part 3: Visual,

http://www.iso.org/iso/iso_catalogue/catalogue_tc/catalogue_detail.htm?csnumber=34230

12. Ivanova, K., Stanchev, P.: Color Harmonies and Contrasts Search in Art Image Collections. First International Conference on Advances in Multimedia (MMEDIA), 20-25.07.2009, Colmar, France, pp. 180-187 (2009)

13. Ivanova, K., Stanchev, P., Dimitrov, B.: Analysis of the Distributions of Color Characteristics in Art Painting Images. Serdica Journal of Computing, Volume 2, Number 2, Sofia, pp. 111-136 (2008) 
14. Amato, G., Gennaro, C., Rabitti, F., Savino, P.: Milos: a Multimedia Content Management System for Digital Library Applications. Research and Advanced Technology for Digital Libraries: Volume 3232/2004 in Lecture Notes in Computer Science, Springer Berlin/Heidelberg, pp. 14-25 (2004)

15. Karypis, G.: CLUTO: A Clustering Toolkit Release 2.1.1. University of Minnesota, Department of Computer Science, Minneapolis, MN 55455, Technical Report: \#02-017 (2003)

16. Arnheim, R.: Art and Visual Perception: A Psychology of the Creative Eye. University of California Press, Berkeley, 1974.

17. Clark, K.: Civilisation - a Personal View. British Broadcasting Corporation, London and John Murray, London. 1969. 